\title{
Low-cost System for Supply Chain Management
}

\author{
K. Fürst, T. Schmidt \\ Institute of Flexible Automation - University of Technology Vienna, Austria \\ Em: kf@infa.tuwien.ac.at
}

Keywords Internet, EDI, XML, Supply Chain Management

\begin{abstract}
In this paper, a complete concept for Internet Electronic Data Interchange (EDI) - a well-known buzzword in the area of logistics and supply chain management to enable the automation of the interactions between companies and their partners - using XML (eXtensible Markup Language) will be proposed. This approach is based on Internet and XML, because the implementation of traditional EDI (e.g. EDIFACT, ANSI X.12) is mostly too costly for small and medium sized enterprises, which want to integrate their suppliers and customers in a supply chain. The paper will also present the results of the implementation of a prototype for such a system, which has been developed for an industrial partner to improve the current situation of parts delivery. The main functions of this system are an early warning system to detect problems during the parts delivery process as early as possible, and a transport following system to pursuit the transportation.
\end{abstract}

\section{INTRODUCTION}

We write the paper to disseminate the results of our industrial project with an engine manufacturer. The aim of the project was the development of a whole new concept for Supply Chain Management to integrate their suppliers and carriers, and the realization of an easy prototype using the latest Internet technologies to evaluate the usefulness of such a system.

The starting point of the project was a problem of our industrial partner (BMW Motors Steyr in Austria). During the process of parts delivery the company has to collect manually a lot of data either from the suppliers or from the carriers. Because this method of data collection is very timeconsuming, it happens only if some problems during this process are becoming visible. The system, which was developed with the engine manufacturer, has to fulfil two main functions: 
- The monitoring of the suppliers and the carriers for increasing the ontime departure performance of parts delivery.

- The responsibility of parts delivery should be transferred to the suppliers. Therefore it is necessary to expose internal logistics data to the suppliers.

In both tasks there is need for a transparent, flexible and low-cost data exchange method, based on the latest Internet technology. The proposed system makes it possible that all relevant data for controlling the process of parts delivery are available automatically and electronically for the industrial partner, all suppliers and carriers of this factory, at any time.

\section{SUPPLY CHAIN MANAGEMENT}

Supply Chain Management (SCM) deals with the management of material, information, and financial flows in a network consisting of vendors, manufacturers, distributors and customers. Managing flows in this network is a major challenge due to the complexity (in space and time) of the network, the proliferation of products (often with short life cycles) that flow through this network, and the presence of multiple decision makers who each own and operate a piece of this network and optimise a private objective function. [11]

Material usually flows from a supplier to a buyer while information and financial flows are bi-directional.

Supply chains today are increasingly depending on effective and efficient information exchanges between the value chain partners. Over the last decade, practices such as just in time management, quick response manufacturing and lean production systems have required coordinated and reliable information exchanges between trading partners. Massive investments in information technology have been made by manufacturers, suppliers and logistics providers with the hope of achieving successful just in time implementation in their supply chains. [11]

In just in time supply chains, the coordination of material flows through information exchange is becoming increasingly important. With technologies such as Electronic Data Interchange (EDI) with suppliers, the rapid transmission of information with trading partners allows manufacturers of efficiently manage logistics activities. [11]

Figure 1 shows the market growth of Supply Chain Management. 


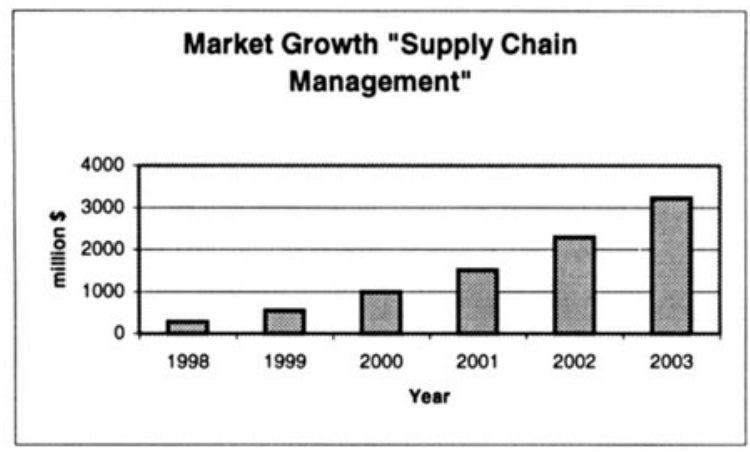

Figure I SCM-Market Growth (Source: IDC 1999)

\section{TRADITIONAL EDI}

Over the past several decades' corporations have invested trillions of dollars in automating their internal processes. While this investment has yielded significant improvements in efficiency, that efficiency has been limited to internal processes. In effect, companies have created islands of automation, which are isolated from their vendors and customers. The interaction between companies and their trading partners remains slow and inefficient, because it is based on manual processes. [6]

Electronic Data Interchange (EDI) has been heralded as the solution to this problem. EDI is defined as the exchange of data between heterogeneous systems to support transactions. This is not simply the exportation of data from one system to another, but the actual interaction between systems. Companies that have implemented EDI rave about the various benefits. In fact, these benefits can be expanded to a chain of suppliers. [6]

There is a significant gap between the business benefits described above and the actual implementation of EDI. This is because the actual implementation of EDI is difficult and costly to implement. More importantly, however, it requires a unique solution for each pair of trading partners. Many people falsely proclaimed the Internet as the solution to this problem. By implementing EDI over a single network, our problems would be solved. Unfortunately, a network with a common protocol is still only a partial solution. This is because the systems implemented in each company are based on different platforms, applications, data formats, protocols, schemas, business rules and more. Simply "connecting" these systems over the Internet does not solve the problem. [6]

Traditional EDI is based on fixed transaction sets. These transaction sets are defined by standards bodies such as the United Nations Standard Messages Directory for Electronic Data Interchange for Administration, 
Commerce and Transport (EDIFACT), and the American National Standards Institute' s (ANSI) Accredited Standards Committee (ASC) X12 sub-group. Transaction sets define the fields, the order of these fields, and the length of the fields. Along with these transaction sets are business rules, which in the lexicon of the EDI folks are referred to as "implementation guidelines". [6]

\section{SYSTEM-ARCHITECTURE}

The system architecture in figure 2 is based on the Internet technology and the new Internet language XML. Because the internal systems of the suppliers and carriers are not equal, it is necessary to extract the relevant data for the new system from these systems and convert the data locally into the international standardized format XML to make it available for the application, the so-called Data-Extractor, which runs on a Web server. The Data-Extractor is responsible for the whole data transmission between the different systems and should handle the requests from the different users working with a standard Web browser. The firewall between the Intranet and the global Internet is necessary to guarantee a high secure-level for the sensible industrial data.

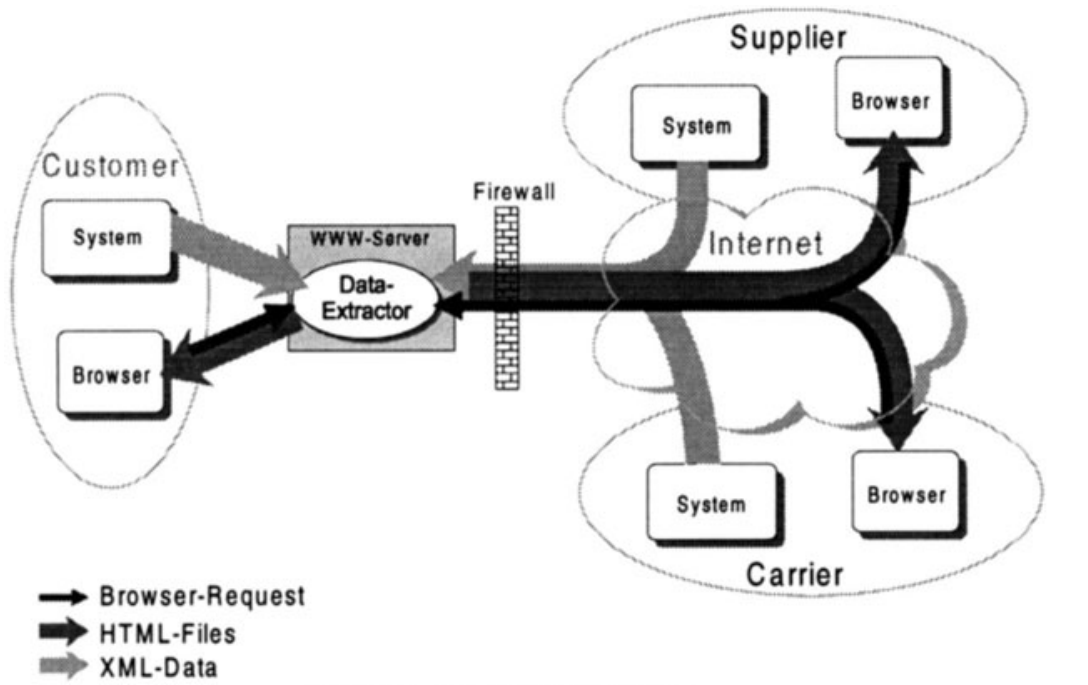

Figure 2 System Architecture

The advantages of this system architecture are:

- Platform independence of this system because the Data-Extractor is implemented with the programming language Java, exactly with Java-Servlets. The format XML is also platform independent. 
- The users can work with a standard Web browser like Microsoft Explorer or Netscape Navigator.

- Using all advantages of the Internet technology like the availability of Internet or the low-cost data transmission over Internet.

The standard for Internet communication in the near future, XML (eXtensible Markup Language), was standardized in the year 1998 by the World Wide Web Consortium and is a subset of SGML (Standard Generalized Markup Language), see figure 3. SGML was standardized in the year 1986 by ISO (International Standards Organization), but it is too complicated and therefore not used in a broad range. With the easier to use language $\mathrm{XML}$ it is also possible to separate data and markup. $\mathrm{XML}$ is a universal data format that allows computers to store and transfer data that can be understood by any other computer system.

See $[1,2,4,6,9]$ for more information about XML.

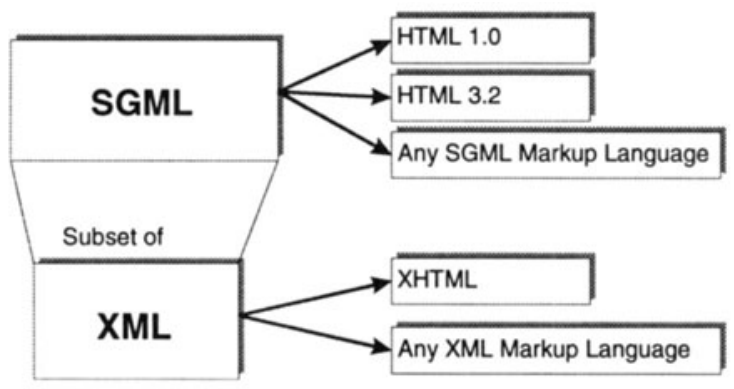

Figure 3 Difference XML - HTML

\section{SYSTEM-FUNCTIONALITY}

Using UML (Unified Modelling Language) the complete system model was designed. Figure 4 shows the simplified Use Case Diagram. In this diagram, the interactions between the following Use Cases are displayed:

- Warning System: With this system the user can manage the whole part delivery, because the output of this Use Case is a table, where the delivery and transport data of all parts are controlled based on the delivery order. If the data differs, the system reports this via a red sign.

- Transport Following: This Use Case reports the actual status of a part transport. The user gets all transport data from a part.

- Part Availability: The user gets information about the actual part stock at BMW and also the delivery and transport data of a part as table with time of delivery and amount. 
- Calling Stock At Supplier: The user gets the actual stock at the supplier in form of a table with the part number, part name and amount.

- Report Part Delivery: It is necessary to report the system the incoming of a delivery (e. g. to update the delivery and transport data).

- Send Delivery Data: The supplier must send the delivery data to BMW.

- Get Delivery Order: The system calls the actual delivery order of a part.

- Get Delivery Data: Calling the actual delivery data of a part, which are stored locally in form of XML-files.

- Get Transport Data: The carrier calls the actual transport data of a part.

- Get Stock At BMW: The user gets the stock at BMW.

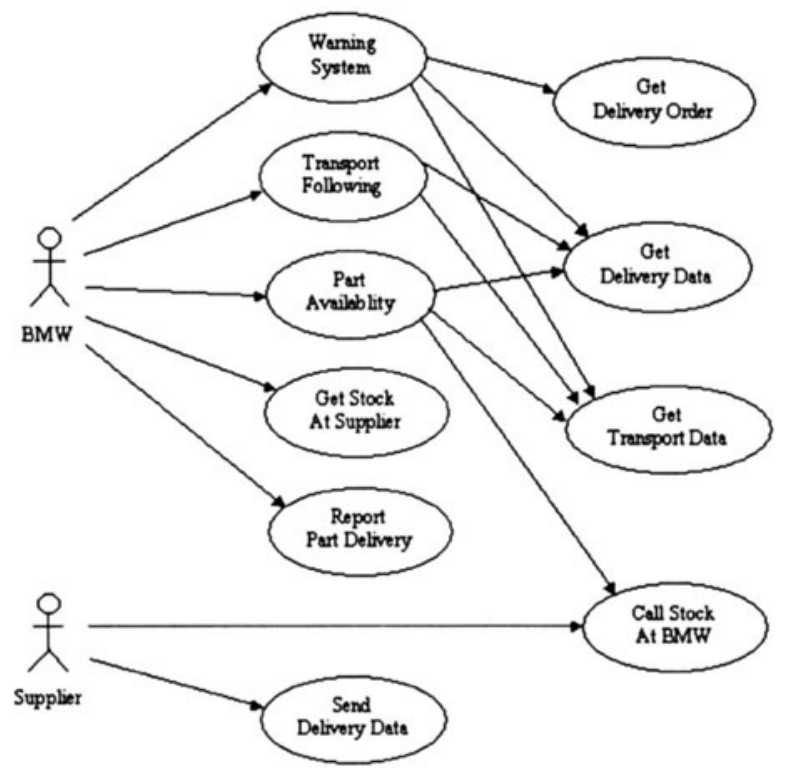

Figure 4 Use-Case-Diagram System-Functionality

\section{IMPLEMENTATION AND TESTING}

The whole application (Data-Extractor) is implemented in Java. Platform independence is the main goal of Java and it has been achieved. Because the application is running on a Web server, Java-Servlets, which realize the functionality of the Use Case Diagram (see figure 4), are used. In general the rise of server-side Java applications is one of the latest and most exciting trends in Java programming. A Java-Servlet is a small, pluggable extension to a server that enhances the functionality of the server and runs inside a Java Virtual Machine on the server, so unlike applets, they do not require support for Java in the Web browser. 
Each servlet has the same life cycle (see figure 5): [12]

- A server loads and initializes the servlet

- The servlet handles zero or more client requests

- The server removes the servlet (some servers do this step only when they shut down)

See $[3,7]$ for more information about Java, and [8, 12] for more information about Java-Servlets.

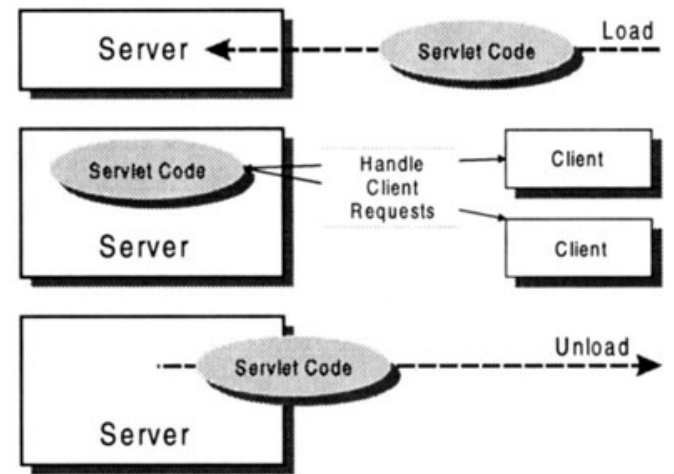

Figure 5 Java-Servlet-Life-Cycle

The Java-Servlets at the Web server handle the GET-Requests of the users (Customer, Suppliers) and work with the XML-Data. For these operations many software tools are developed since the standardization of XML. Under the developers are companies like JavaSoft, Microsoft, Oracle and IBM. This show how important the combination of Java/XML will be in the near future.

There are two major types of XML-APIs: $[2,10]$

- A tree-based API compiles an XML document into an internal tree structure and allows an application to navigate that tree. The DOM (Document Object Model) is such an API, which is in the process of being standardized by W3C.

- An event-based API, on the other hand, reports parsing events (such as the start and end of elements) to an application through callbacks. The application implements handlers to deal with the various events. SAX (Simple API for XML) is such an API.

The servlets in the prototype use the DOM interface to operate with the XML data. After parsing the XML file (figure 6), you can step through the internal tree structure (figure 7) and save the necessary data into internal variables.

After working with this internal data (e.g. calculate the Warning System under using a special logic), the servlet generate a HTML-Output and send 
this to the client. The user can see this output using a normal Web browser like Microsoft Explorer or Netscape Navigator.

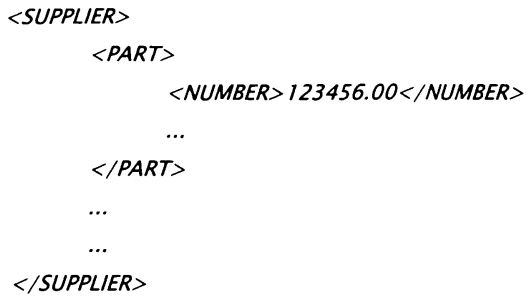

Figure 6 XML-File

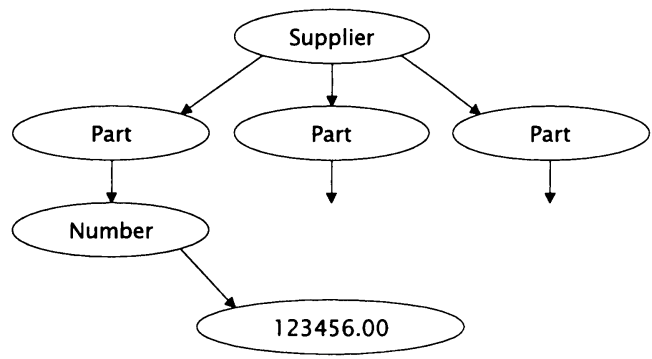

Figure 7 DOM

\section{CONCLUSIONS}

The results of testing the prototype give us the motivation to implement the next step of the project - the connections to the different in-house systems. Because the users of this prototype (supplier and carrier) didn't want to install additional XML-EDI software for prototype testing, we realized the necessary integration with HTML-forms, which will be filled out manually by the users. Only the interface to the internal SAP-System will be realized to work automatically. The realized prototype can work with dynamical, real-time data. After the prototype test-phase, our industrial partner can quantify the advantages of such a new, low-cost system for electronic data interchange and supply chain management using the latest Internet technologies XML and Java.

\section{REFERENCES}

[1] Behme H. (1999). Kunst der Stunde, IX-Magazin für professionelle Informationstechnik, 2/99, 36-41

[2] Chang D., Harkey D. (1998). Client/Server Data Access with Java and XML, Wiley Computer Publishing

[3] Flanagan D. (1996). Java in a nutshell, O'Reilly

[4] Goldfarb C.F., Prescod P. (1999). XML-Handbuch, Prentice-Hall

[5] Helios, http://www.wiinf.uni-wuerzburg.de/helios/startseite-main.htm (27.5.1999)

[6] Hogan M. (1998). XML and the Internet: Driving the future of EDI, POET-Software

[7] Huber G. (1996). Java - Die Referenz, Heise

[8] Hunter J. (1998). Java Servlet Programming, O'Reilly

[9] John V. (1999). XML - Weltsprache für das Internet, OBJEKTspektrum, 5/99, 22-28

[10] Middendorf S. (1999). XML und Java, OBJEKTspektrum, 5/99, 38-40

[11] Servlets-Tutorial, http://www.uni-klu.ac.at/unihome/header/tutorial/servlets/ (10.9.1999)

[12] Tayur S., Ganeshar R., Magazine M. (1999). Quantitative Models for Supply Chain Management, Kluwer Academic Publishers 